\title{
Mitteilung
}

\section{Organisationsverschulden in Klinik und Praxis}

\section{Ärztliche Standards bei strukturellen und organisatori- schen Veränderungen}

\author{
Albrecht Wienke ${ }^{1}$ \\ ${ }^{1}$ Deutsche Gesellschaft für Medizinrecht (DGMR) \\ GMS Mitteilungen aus der AWMF 2006;3:Doc03
}

\begin{abstract}
Zusammenfassung
Einbecker-Empfehlungen der Deutschen Gesellschaft für Medizinrecht (DGMR) e.V.
\end{abstract}

Die Deutsche Gesellschaft für Medizinrecht (DGMR) e.V. hat gemeinsam mit der Kaiserin-Friedrich-Stiftung für das ärztliche Fortbildungswesen am 02. und 03. Dezember 2005 das 31. Symposion für Juristen und Ärzte veranstaltet. Als Ergebnis dieser Tagung wurden die nachstehenden Empfehlungen verabschiedet:

\section{Text}

\section{Problemstellung}

Die Verrechtlichung und Ökonomisierung der ärztlichen Tätigkeit in Klinik und Praxis schreitet mit zunehmender Geschwindigkeit voran. Einerseits zwingen beschränkte wirtschaftliche und personelle Ressourcen zu Reglementierungen der ärztlichen Berufsausübung; anderseits werden ständig neue Qualitätsanforderungen gestellt. In dieser Zwickmühle sehen sich die Ärzte in Klinik und Praxis neuerdings weiteren Herausforderungen ausgesetzt, die auf gesetzlich und ökonomisch bedingte Strukturund Organisationsveränderungen zurückgehen. Auch die Verantwortung für eine sachgemäße Organisation der ärztlichen Behandlungsabläufe unterliegt derzeit vielfältigen Neuordnungen. Modulares Großkrankenhaus, Medizinische Versorgungszentren (MVZ), integrierte Kooperationsstrukturen zwischen ambulanten und stationären Behandlungseinheiten, fachgebietsübergreifende Dienste, Behandlungspfade, Scoresysteme und die Zentralisierung von Behandlungseinheiten sind Beispiele neuer Strukturen und Organisationsformen im Gesundheitswesen, mit denen die ärztliche Diagnostik und Therapie und das vertrauensvolle Arzt-Patienten-Verhältnis in Wettbewerb stehen. Handlungsund Therapiefreiheit der Ärzte in Klinik und Praxis sind berührt; eine Erhöhung der Risiken für die Patienten und ein erhöhtes Risiko juristischer Haftung wird befürchtet.

Diese tiefgreifenden strukturellen und organisatorischen Veränderungen im Medizinbetrieb erfordern kontinuierlich eine juristische Neubewertung.

\section{Gegenwärtiges rechtliches Verständnis des Organisationsverschuldens}

Das Organisationsverschulden bildet eine zentrale Kategorie des modernen Zurechnungs- und Haftungsrechts. Gerade im klinischen Altag gewinnen Organisationspflichten immer mehr an Gewicht. Je größer die Zahl der an Diagnose und Therapie beteiligten Ärzte, Techniker und Hilfskräfte, je kompli-

\footnotetext{
${ }^{1}$ Corresponding Author:

Dr. iur. Albrecht Wienke, Generalsekretär der DGMR, Bonner Str. 323, 50968 Köln
} 
zierter und gefährlicher die apparativen und medikamentösen Mittel, je komplexer das arbeitsteilige medizinische Geschehen in einem großen Betrieb und je größer der ökonomische Erfolgsdruck, desto mehr Umsicht und Einsatz erfordern die Planung, die Koordination und die Kontrolle der klinischen Abläufe.

\section{Gesetzliche Anknüpfungspunkte des Organisationsverschuldens}

Auswahl-, Überwachungs- und Anleitungspflichten bilden zusammen mit der Pflicht zur Ausstattung des ärztlichen und pflegerischen Personals mit adäquaten technischen Hilfsmitteln einen umfangreichen Pflichtenkatalog des Krankenhausträgers und Praxisinhabers. Dieser umfasst auch ausreichende personelle und infrastrukturelle Ressourcen (z.B. IT- Ausstattung und Festlegung von Handlungsanleitungen).

Krankenhäuser oder in der Form der juristischen Person betriebene Arztpraxen sind für den Schaden verantwortlich, den ein leitender Mitarbeiter (Chefarzt, Oberarzt) einem Patienten zufügt. Primär verantwortlich ist daher der Träger der jeweiligen medizinischen Einrichtung. Eine Verantwortung der leitenden Mitarbeiter im Übrigen kommt in Betracht, wenn der Träger nachweisen kann, dass er die ihm obliegenden Organisationspflichten wahrgenommen hat, sog. Entlastungsbeweis i. S. v. § 831 Abs. 1 Satz 2 BGB. Es genügt für eine solche Haftungsfreistellung allerdings nicht, dass der Träger den Nachweis sorgfältiger Auswahl und Überwachung des von ihm eingesetzten, für den fraglichen Bereich verantwortlichen höheren Angestellten führt. Vielmehr trifft ihn darüber hinaus die Pflicht, den gesamten Ablauf der Betriebsvorgänge und die Tätigkeit des Personals durch geeignete organisatorische Vorkehrungen so einzurichten und zu überwachen, dass Dritte nicht geschädigt werden.

\section{Prozessuale Anknüpfungspunkte}

Die Rechtsprechung geht bereits dann von einem pflichtwidrigen Verhalten des Krankenhauspersonals aus, wenn feststeht, dass der Patient „im Organisationsbereich des Krankenhauses“ zu Schaden gekommen ist (z.B. durch Transport, unsterile Infusionsflüssigkeit oder defekte medizinische Apparaturen). Wie im Produkthaftungsrecht wird von einem objektiven Mangel oder verkehrswidrigen Zustand auf ein pflichtwidriges Verhalten geschlossen.

\section{Organisationspflichten in der Rechtsprechung}

Folgende Kardinalpflichten lassen sich der Rechtsprechung entnehmen:

(1) Zuständigkeiten und Verantwortlichkeiten im Krankenhaus müssen durch Einsatzpläne und Vertreterregelungen deutlich abgegrenzt und insbesondere Sonntags-, Nacht- und Bereitschaftsdienste gesichert sein. Auch für die Patientenaufklärung und die ärztliche Erstversorgung von Unfallopfern bedarf es besonderer Anweisungen.

(2) Die ärztlichen und die nicht-ärztlichen Mitarbeiter müssen sorgfältig ausgewählt, angelernt und überwacht werden. In jeder Behandlungsphase muss ein qualifizierter Arzt bereit stehen, um die erforderlichen Maßnahmen durchzuführen, zu delegieren und zu überwachen.

(3) Das Krankenhaus muss die ärztlichen und pflegerischen Standards in personeller, fachlicher und apparativer Hinsicht gewährleisten.

(4) Die Sicherheit der Patienten muss gewährleistet sein. Besonderen Anfälligkeiten von Kindern oder von verletzungs- oder suizidgefährdeten Personen ist Rechnung zu tragen. Darüber hinaus müssen die hygienischen Verhältnisse und die Funktionsfähigkeit aller medizinischen Geräte und Apparate sichergestellt sein.

\section{Veränderung der äußeren Rahmenbedingungen}

Die Organisationspflichten in Klinik und Praxis werden zunehmend von folgenden geänderten äußeren Rahmenbedingungen bestimmt:

1. Die demografische Entwicklung und die Fortschrittsfalle in der Medizin erfordern ein gemeinsames, koordiniertes Vorgehen derer, die Verantwortung für das Gesundheitswesen tragen. Ohne geeignete Anpassung des Krankenversicherungssystems an die feststellbaren Veränderungen und voraussehbaren Anforderungen ist die Versorgung der Patienten gefährdet. 
2. Aktuelle Erkenntnisse zur zukünftigen Arztzahlentwicklung belegen, dass die sich bereits jetzt abzeichnende Personalverknappung im ärztlichen Dienst in den kommenden Jahren zunehmen wird.

3. Durch eine stärkere Leistungsverdichtung wird sich der Anteil der sog. „sprechenden Medizin" weiter verringern - mit negativen Konsequenzen für die individuelle Arzt-PatientenBeziehung. Die seit Jahren zunehmenden bürokratischen Anforderungen im Klinik- und Praxisalltag verschlechtern diese Situation zusehends.

4. Auch die diskutierte Einführung der Bachelor- und Master-Studiengänge führt zu heterogenen Qualifikationsmaßstäben in der medizinischen Versorgung, die haftungsrechtliche Konsequenzen nach sich ziehen kann. Dies gilt auch für die zunehmende Übertragung ärztlicher Aufgaben auf nicht-ärztliche Berufe.

5. Das Missverhältnis zwischen leistungsrechtlichem Versorgungsanspruch und der erforderlichen Finanzausstattung der gesetzlichen Krankenversicherung ist trotz der bisherigen Reformversuche eine weiter bestehende Herausforderung.

6. Das Krankenhausvergütungssystem der diagnosebezogenen Fallpauschalen (DRG) führt zu neuen Konfliktlagen zwischen betriebswirtschaftlichen und medizinischen Gesichtspunkten der Krankenbehandlung.

7. Die von den medizinisch-wissenschaftlichen Fachgesellschaften entwickelten Leitlinien werden zunehmend nicht mehr nur als Handlungskorridor für den behandelnden Arzt im Einzelfall verstanden, sondern in Form von Behandlungsschemata und -pfaden sowie Versorgungsanweisungen zur „Programmmedizin“.

\section{Struktur- und Organisationsveränderungen in Klinik und Praxis}

Als Reaktion auf diese geänderten äußeren Rahmenbedingungen in Klinik und Praxis seien folgende Beispiele genannt:

\section{Interdisziplinäre Behandlungseinheiten im modularen Großklinikum}

Es gibt Bestrebungen, die bisherige Struktur des Krankenhauses in Form der Zuordnung der Patienten nach bestimmten Krankheiten zu fachgebietsbezogenen Abteilungen des Krankenhauses durch ein modulares Krankenhaus zu ersetzen, in dem Patienten scoregesteuert entsprechend dem jeweiligen Schweregrad des Behandlungsgeschehen in interdisziplinären Funktionskliniken mit den Bereichen Intensive Care, Intermediate Care und Standard-Care behandelt werden. Die Klinikdirektoren der einzelnen operativen Fachkliniken sollen dort für die Versorgung der Patienten in den interdisziplinären Bereichen schriftlich vereinbarte Behandlungsschemata entwickeln, nach denen eine gleichförmige und qualitätsgesicherte Patientenversorgung im Standardverfahren erfolgen soll.

Den damit verbundenen Veränderungen der Aufteilung von Verantwortung und Risiken ist durch vorausschauende Konzeption zu begegnen, neu entstehende Risiken sind zu neutralisieren. Als solche Risiken müssen beispielsweise die Unterberechung der persönlichen Verantwortungskontinuität und Kommunikationsdefizite gelten. Gerade im Hinblick auf die Interdisziplinarität dieser Versorgungsstruktur ist der Facharztstandard einzuhalten.

Geeignete Risikomanagement-Verfahren sollten die Einführung veränderter Strukturen und Organisationsformen stets begleiten.

Soweit Ärzte in solchen Versorgungsformen die Voraussetzungen für eine Behandlung nach dem anerkannten Stand der medizinischen Wissenschaft nicht mehr gewährleistet sehen, können sie in schwerwiegende Konflikte zwischen berufsrechtlichen und ethischen Verpflichtungen einerseits und arbeitsrechtliche und dienstrechtliche Konsequenzen andererseits geraten. In derartigen Situationen ist eine patientenorientierte Konfliktauflösung zugunsten der ärztlichen Therapiefreiheit geboten.

Gleiches gilt für die teilweise bereits eingeführten fachgebietsübergreifenden Bereitschaftsdienste. Auch für diese ist der Facharztstandard sicherzustellen.

\section{Schichtdienste}

Die Anwendung des Arbeitszeitgesetzes auf den Medizinbetrieb führt in einigen Bereichen zwangsläufig zu Schichtdiensten. Die Einordnung des Bereitschaftsdienstes als Arbeitszeit hat darüber hinaus zur Folge, dass die gesetzlichen Maximalarbeitszeiten früher erreicht werden als bisher. Dies bedingt einen höheren Bedarf an verfügbarem Personal, vermehrte Anzahl von Übergaben mit der 
damit zwangsläufig erhöhten Gefahr von damit verbundenen Kommunikationsdefiziten. Es sind organisatorische Maßnahmen zu treffen, welche diese Risiken ausschalten.

\section{MVZ und integrierte Versorgung}

Der Betreiber eines medizinischen Versorgungszentrums (MVZ) hat durch gezieltes Risikomanagement und Maßnahmen der Qualitätssicherung die Möglichkeit, die sogenannten „Schnittstellen“ im Rahmen der arbeitsteiligen Medizin miteinander zu verzahnen und damit die Kommunikation und Kooperation zwischen ambulanter und stationärer Versorgung zu gewährleisten. Ein einheitliches Risikomanagement zwischen medizinischem Versorgungszentrum und Krankenhaus bei demselben Betreiber steigert die Effizienz in der Krankenversorgung, vermeidet unnötige Kontroll- und Mitarbeitspflichten und schützt die Patienten davor, dass sie durch Zuständigkeits- oder Informationslücken Schaden erleiden oder dadurch, dass ein an der Behandlung Beteiligter notwendige Maßnahmen in der unbegründeten Vermutung unterlässt, ein anderer werde schon das Nötige veranlasst haben. Diese Anforderungen an die haftungsrechtliche Koordination müssen auch für die poststationäre Versorgung gelten.

In der integrierten Versorgung nach $\S 140 \mathrm{~b}$ SGB $\vee$ bestehen ähnliche Risiken der Haftung und Qualitätssicherung wie beim MVZ. Das Ziel der integrierten Versorgung, die Trennung der einzelnen Leistungsbereiche zu überwinden, Substitutionsmöglichkeiten über die verschiedenen Leistungssektoren hinweg zu nutzen und Schnittstellenprobleme zu beherrschen, wird befördert, wenn die Qualitätssicherungsmaßnahmen Bestandteil des Integrationsvertrages werden. Hierzu kann ein verbindliches Behandlungsmanual dienen, in dem strukturierte Versorgungsabläufe, Behandlungspfade, Leitlinien und Standards, Basisdokumentationen und Qualitätsindikatoren für die Struktur- und Prozessqualität vertraglich fixiert werden, ohne allerdings die individuelle Behandlungsfreiheit und -verantwortung des Arztes zu ersetzen. Diese Qualitätssicherungsmaßnahmen - wie die Entwicklung projektbezogener Dokumentationsformulare, die Schulung von externen Partnern und die Validierung der dokumentierten Daten - können allerdings nur dann das Ziel erreichen, wenn sich die einzelnen Beteiligten innerhalb der integrierten Versorgung mit den Chancen dieser neuen Versorgungsformen identifizieren.

\section{Verstärkung der Arzt - Patienten - Beziehung}

Um Gefahren in organisatorischer Hinsicht bei Einführung solcher oder ähnlicher Organisationsstrukturen von Patienten abzuwenden, muss eine ausreichende personelle Besetzung mit genügend Zeit für Gespräche und Behandlung zur Verfügung gestellt werden. Ein Patient will von verantwortungsbewussten und vertrauten Ärzten und Pflegepersonal behandelt werden, und nicht in einen anonymen, artifiziellen und gleichförmigen Behandlungsprozess eingebunden sein. Maßnahmen hierfür könnten z.B. sein ein critical incident reporting system (CIRS), um vorhersehbare Fehler zu vermeiden und Leitlinien in sachgerechter Anwendung einzuführen. Das aus personellen und finanziellen Einschränkungen sich ergebende Haftungsrisiko im Sinne eines Organisationsverschuldens darf nicht ausschließlich den vor Ort verantwortlichen Ärzten überlassen bleiben. Vielmehr ist es notwendig, den jeweiligen Träger eines Krankenhauses verstärkt in die sächliche Organisationsverantwortung einzubinden.

\section{Rechtliche Bewertung}

\section{Zivilrechtlicher Sorgfaltsmaßstab}

Eine Anpassung des zivilrechtlichen Sorgfaltsmaßstabs im formellen Sinne an eingetretene Veränderungen ist nicht erforderlich. Die Gesetzeslage lässt der Rechtsprechung genügend Raum, die Kriterien festzulegen, nach denen ein Pflichtenverstoß anzunehmen und die im Verkehr erforderliche Sorgfalt gemäß § 276 Abs. 2 BGB nicht beachtet ist, wobei Veränderungen der tatsächlichen Umstände oder anderweitig verfügter Pflichtenkataloge unschwer berücksichtigt werden können. Haftungsrechtlich wird das zu beurteilende Tun oder Unterlassen unverändert daran gemessen, ob die im Verkehr erforderliche Sorgfalt gewahrt ist. Auch definitorisch bleibt der rechtliche Sorgfaltsmaßstab unverändert. Anknüpfungspunkt für die verkehrserforderliche Sorgfalt der an der Behandlung beteiligten Ärzte ist (und bleibt) der medizinische Standard des betroffenen Fachgebiets, wobei der objektiv-typisierende Maßstab gilt.

In der Rechtsordnung gilt der Grundsatz der Deckungsgleichheit des rechtlichen und des medizinischen Sorgfaltsmaßstabs. Dieser Grundsatz erfährt aber nur dann eine Ausnahme, als es das 
Haftungsrecht unbeanstandet lässt, wenn die konkrete Behandlung hinter dem Stand der medizinischnaturwissenschaftlichen Erkenntnisse zurückbleibt, weil Grenzen der Finanzierbarkeit oder der Wirtschaftlichkeit dies bedingen. Dies kann dann Auswirkungen auf den zu fordernden Standard haben. Allerdings darf in keinem Fall der Bereich des medizinisch zwingend Notwendigen verletzt werden. Dies gilt ebenso für den Fall, dass sich die diskutierten strukturellen und organisatorischen Veränderungen im Medizinbetrieb tatsächlich in nennenswertem Umfang einstellen sollten. Diese dürfen den medizinischen Standard beeinflussen, jedoch nicht zu einer Unterschreitung der unverzichtbaren Basisschwelle führen. Schließlich ist es auch dem Sozialgesetzgeber verwehrt, in diesen Kernbereich des medizinisch zwingend Notwendigen regelnd (z.B. durch DMP - disease management program) einzugreifen. Dies kann haftungsrechtlich nicht akzeptiert werden und würde einer verfassungsrechtlichen Prüfung wegen Verstoßes gegen das Sozialstaatsprinzip nicht standhalten.

\section{Haftung leitender Ärzte}

Forderungen nach einer Beschränkung der Außenhaftung angestellter Krankenhausärzte werden auch hierzulande zunehmend erhoben. Dies sei angezeigt zum Zwecke einer Vereinfachung der überaus komplizierten Haftungsverhältnisse im Krankenhaus, zumal für die Schadenskompensation die Einstandspflicht des Trägers völlig ausreiche. Eine derartige Regelung führte zu einem haftungsrechtlichen Rückzug vor allem leitender Ärzte hinter die Anonymität des Klinikträgers. Ärztliches Ethos, Berufs- und Standesrecht und das Vertrauensverhältnis zwischen Arzt und Patient weisen jedoch auf eine strenge persönliche Haftung des Arztes hin. Die Vorschläge einer Beschränkung der Außenhaftung verdienen daher keine Unterstützung.

\section{Strafrechtliche Beurteilung}

Bei der strafrechtlichen Beurteilung des Organisationsverschuldens gelten zum objektiven Sorgfaltsmaßstab zunächst dieselben Kriterien wie bei der zivilrechtlichen Betrachtung. Eine Verantwortlichkeit wird daher angenommen, wenn der Patient im Organisationsbereich des Krankenhauses zu Schaden kommt. Im Gegensatz zur zivilrechtlichen Bewertung ist bei der strafrechtlichen Bewertung des Organisationsverschuldens darüber hinaus auch der subjektive Sorgfaltsmaßstab zu berücksichtigen. Dieser orientiert sich neben der dem Einzelnen möglichen Erkennbarkeit der Gefahr an der tatsächlichen Fähigkeit, die Gefahr abzuwenden, d.h. an der persönlichen Vermeidbarkeit eines schadensverursachenden Organisationsfehlers. Die bei der Realisierung eines Organisationsrisikos besonders relevante Übernahmefahrlässigkeit wird durch die Frage der Vorwerfbarkeit begrenzt.

Wenn das Sozialrecht über die Finanzierung dem ärztlichen Handeln gewisse Grenzen setzt, so kann die Übernahme eines von der Allgemeinheit - durch das Sozialrecht in Gesetzesbuchstaben formuliert - erwünschten Verhaltens innerhalb dieser Grenzen nicht als vorwerfbar angesehen werden. Daneben kann ein derartiges Verhalten als innerhalb der Sozialadäquanz liegend und damit als nicht strafbar gelten.

Wie das Bundesverfassungsgericht festgestellt hat, ist die Sicherung der finanziellen Stabilität der Krankenversicherung ein Gemeinwohlbelang von überragendem Gewicht. Sie dient insgesamt der Aufrechterhaltung eines funktionierenden Gesundheitssystems und damit der Gesundheit aller Versicherten. Es bietet sich daher an, die finanziellen Rahmenbedingungen, die sich den Leistungserbringern im Gesundheitssystem darstellen, dem Prinzip des überwiegenden Interesses im Rahmen des erlaubten Risikos zu unterschreiben. Dies scheint insbesondere deshalb geboten, weil sich damit Wertungswidersprüche zwischen den einzelnen Sparten der Rechtsordnung, hier dem Haftungs- bzw. Strafrecht und dem Sozialrecht auflösen lassen.

Bei der Standardunterschreitung liegt der Schwerpunkt der Vorwerfbarkeit auf dem Unterlassen des gebotenen Handelns. Die Erfolgsabwendungspflicht des Garanten, d.h. die Pflicht des Arztes, das medizinisch Erforderliche zu tun, wird durch die Zumutbarkeit und Möglichkeit begrenzt. Es ist zu bedenken, dass dem einzelnen Arzt häufig nur die Alternative bliebe, die medizinische Versorgung unter den (den Standard unterschreitenden) Gegebenheiten gänzlich abzulehnen. Hierdurch kann aber die Gefahr für die zu schützenden Rechtsgüter wachsen, weil z.B. der Weg zur nächsten alternativen Behandlungseinheit ein nicht mehr hinzunehmendes Maß erreicht oder neue weitere Risiken eröffnet 
Wienke: Organisationsverschulden in Klinik und Praxis; GMS Mitteilungen aus der AWMF 2006;3:Doc03

\section{Medizinische Sachverständige}

Die medizinischen Sachverständigen sollten bei der gutachtlichen Bewertung einzelner Behandlungsgeschehen die veränderten äußeren Rahmenbedingungen und die geänderten Versorgungsstrukturen sowie neue organisatorische Behandlungsabläufe zukünftig stärken berücksichtigen.

Berlin im Dezember 2005

Das Präsidium der DGMR e.V. 